\title{
MicroRNA-200b suppresses cell invasion and metastasis by inhibiting the epithelial-mesenchymal transition in cervical carcinoma
}

\author{
YAN-XIANG CHENG $^{1 *}$, QI-FAN ZHANG ${ }^{1 *}$, LI HONG $^{1}$, FENG PAN $^{2}$, \\ JIN-LING HUANG ${ }^{1}$, BING-SHU LI ${ }^{1}$ and MIN HU ${ }^{1}$ \\ Departments of ${ }^{1}$ Obstetrics and Gynecology and ${ }^{2}$ Orthopaedics, Renmin Hospital of Wuhan University, \\ Wuhan, Hubei 430060, P.R. China
}

Received April 5, 2015; Accepted January 8, 2016

DOI: $10.3892 / \mathrm{mmr} .2016 .4911$

\begin{abstract}
The expression of microRNA (miR)-200b is suppressed in numerous tumor types, leading to epithelial-mesenchymal transition, which enables solid tissue epithelial cancers to invade and metastasize. The present study assessed the role of miR-200b in cervical cancer with the aim of clarifying the underlying pathophysiological mechanisms and to identify potential strategies for its prevention and treatment of cervical cancer. Reverse-transcription quantitative PCR revealed that miR-200b was downregulated in invasive cervical carcinoma tissues compared with that in normal adjacent tissues. A Transwell migration assay indicated that transfection of cervical cancer cells with miR-200b mimics significantly inhibited their migratory potential, while migration was enhanced in cells transfected with miR-200b inhibitor. Furthermore, western blot analysis indicated a negative correlation between miR-200b and mesenchymal marker vimentin as well as matrix metalloproteinase-9, which has a key role in tumor invasion and metastasis. In addition, a positive correlation between miR-200b and the epithelial marker E-cadherin was revealed by western blot and immunofluorescence. The results of the present study suggested that miR-200b suppressed the migratory potential of cervical carcinoma cells and therefore their ability to metastasize by inhibiting the epithelial-mesenchymal transition, which may be utilized for the treatment of cervical cancer.
\end{abstract}

Correspondence to: $\mathrm{Dr} \mathrm{Li}$ Hong, Department of Obstetrics and Gynecology, Renmin Hospital of Wuhan University, 99 Zhangzhidong Road, Wuhan, Hubei 430060, P.R. China E-mail: drhongli77@gmail.com

*Contributed equally

Key words: epithelial-mesenchymal transition, microRNA-200b, cervical cancer

\section{Introduction}

Cervical cancer is the second leading cause of all cancer-associated mortalities in females after breast cancer (1). As carcinogenesis is a complex biological process involving a large variety of factors and comprising multiple steps, it is necessary to enhance the current understanding of the mechanisms involved in the spread of carcinomas to identify novel therapeutic targets. MicroRNAs (miRs) are small non-coding RNAs that are 18-24 nucleotides (nt) in length and regulate gene expression at the post-transcriptional level (2). miRs have been implicated in the regulation of tissue morphogenesis and cellular differentiation, and direct link between miRNAs and the pathology of various diseases has been identified, including the occurrence, development, invasion and metastasis of tumors (3-5).

The members of the miR-200 family have been reported to have various functions (6), among which the downregulation of tumor progression and inhibition of epithelial-mesenchymal transition (EMT) are prominent $(7,8)$. The anti-tumor effects of miR-200 include repression of cancer stem cell self-renewal (9), inhibition of cell division (10) as well as induction of apoptosis $(11,12)$. Mounting evidence has indicated that the EMT is a means by which solid tissue epithelial cancers invade and metastasize (13-15). Neoplastic cells undergoing EMT are characterized by an increased potential to invade surrounding tissues and disseminate to distant sites $(16,17)$. During EMT, epithelial cells lose phenotypic features, including apical-basal polarity and cell-cell contacts, and acquire mesenchymal cell properties such as increased cell mobility (18). The EMT is characterized by downregulation of epithelial markers, such as E-cadherin, which has a central role in maintaining normal epithelial morphology, and gain of mesenchymal markers, including fibronectin, vimentin and $\mathrm{N}$-cadherin, accompanied by a loss of cellular polarity (19). The expression of miR-200b has been found to be suppressed in numerous tumor types and, of note, miR-200b is a suppressor of the EMT (20).

The present study assessed the role of miR-200b in cervical cancer, focusing on its effects on the EMT and migration. It was demonstrated that miR-200b acts as a tumor suppressor gene in cervical cancer, and upregulation of miR-200b repressed 
the migration and progression of EMT. The present study contributed to the present understanding of the pathogenesis of cervical cancer and provided an experimental and theoretical basis for the treatment and inhibition of metastasization of cervical cancer.

\section{Materials and methods}

Ethics statement. Human samples used in the present study were obtained according to the principles of the Declaration of Helsinki, and all procedures were approved by the Ethics Committee of the Renmin Hospital of Wuhan University (Wuhan, China). Written informed consent was obtained from all patients.

Clinical specimens. The study cohort consisted of 13 patients with invasive carcinoma of the cervix (ICC) at the early stage (termed stage IA-IIA) identified by pathological diagnosis (age range, 29-66 years; mean age, 46 years), who were treated at Renmin Hospital of Wuhan University (Wuhan, China) between February and July 2014. A total of 13 cervical cancer tissues and 12 normal cervical epithelial tissues were collected at the same time points. Staging was performed according to the standard of the International Federation of Gynecology and Obstetrics (FIGO) (21).

Cell transfection. The human cervical carcinoma cell line HeLa was purchased from the Type Culture Collection of the Chinese Academy of Sciences (Shanghai, China). HeLa cells were cultured in RPMI 1640 medium (Thermo Fisher Scientific, Inc., Waltham, MA, USA) supplemented with $10 \%$ fetal bovine serum (Gibco; Thermo Fisher Scientific, Inc.), $100 \mathrm{U} / \mathrm{ml}$ penicillin and $100 \mu \mathrm{g} / \mathrm{ml}$ streptomycin (Gibco; Thermo Fisher Scientific, Inc.) and cultured in an incubator at $37^{\circ} \mathrm{C}$ with $5 \% \mathrm{CO}_{2}$. The cells were then seeded into six-well plates and grown overnight to reach $\sim 40 \%$ confluence. Cells were transfected with miR-200b mimics (Ribobio, Guangzhou, China), miR-200b inhibitors (Ribobio) and negative control miR (Ribobio) using Lipofectamine 2000 (Invitrogen, Thermo Fisher Scientific, Inc.) in Opti-MEM (Invitrogen) according to the manufacturer's protocol. After $6 \mathrm{~h}$ of transfection, the medium (DMEM; Invitrogen) was removed and replaced with complete growth medium. At $48 \mathrm{~h}$ after transfection, cells were harvested for RNA or protein analysis.

Reverse-transcription quantitative polymerase chain reaction $(R T-q P C R)$. Total RNA was extracted with TRIzol regent (Invitrogen) in accordance with the manufacturer's protocol. For analysis of miR-200b expression, cDNA was synthesized using specific reverse transcription primers (miR-200b, F 5'-GCGGCTAATACTGCCTGGTAA-3'; R 5'-GTGCAG GGTCCGAGGT-3'; U6, F 5'-CGCTTCGGCAGCACATAT ACTA-3'; R 5'-CGCTTCACGAATTTGCGTGTCA-3'). purchased from Ribobio and the miRNA First-strand cDNA Synthesis kit (Fermentas, Vilnius, Lithuania). Real-time PCR was performed in the Step One Plus Real-Time system (Applied Biosystems, Thermo Fisher Scientific, Inc.) with ABI SYBR Green Master Mix (Applied Biosystems), and U6 was used as the endogenous reference gene. The thermocycling conditions were as follows: $95^{\circ} \mathrm{C}$ for $10 \mathrm{~min}$, followed by
40 cycles of $95^{\circ} \mathrm{C}$ for $15 \mathrm{sec}$ and $60^{\circ} \mathrm{C}$ for $1 \mathrm{~min}$. The gene expression levels were calculated relative to the expression of U6 using the $2^{-\Delta \Delta \mathrm{Cq}}$ method (22).

Cell migration assays. Cell migration was examined by a Transwell migration assay. Transwell insert chambers with $8-\mu \mathrm{m}$ porous membranes (Corning Inc., Corning, NY, USA) were used. Following $24 \mathrm{~h}$ of transfection, the cells were dislodged from the culture vessel surface with trypsin (Gibco, Thermo Fisher Scientific, Inc.) suspended at $2 \times 10^{5}$ cells $/ \mathrm{ml}$. For each group, $200 \mu \mathrm{l}$ cell suspension was seeded into the upper Transwell chambers. The lower compartment contained $10 \%$ fetal calf serum (Gibco). After $24 \mathrm{~h}$ of incubation, cells on the upper side of the membrane were removed, followed by washing with phosphate-buffered saline (PBS) three times. The migrated cells on the bottom side of the membrane were then fixed with methanol (Goodbio Technology, Wuhan, China) for $15 \mathrm{~min}$ at room temperature and stained with $0.5 \%$ crystal violet (Sigma-Aldrich, St. Louis, MO, USA) in $25 \%$ methanol for $5 \mathrm{~min}$. Images of migrated cells in at least six random selected fields were captured under a microscope at 100x magnification and cells were counted. Experiments were performed in technical and biological triplicates. One representative high-power field per Transwell membrane was counted (using six randomly selected high-power fields per treatment condition).

Western blot analysis. Following transfection, cells were lysed in radioimmunoprecipitation assay buffer (Beyotime Institute of Biotechnology, Haimen, China). The obtained protein extracts were centrifuged at $16,099 \mathrm{x} g$ for $15 \mathrm{~min}$. The protein concentrations were determined using a bicinchoninic acid protein assay kit (Beyotime Institute of Biotechnology). For western blot analysis, equal quantity $(30 \mu \mathrm{g})$ of protein was loaded in each lane and subjected to $10 \%$ sodium dodecyl sulfate polyacrylamide gel electrophoresis. Proteins were then transferred onto a polyvinylidene difluoride membrane (EMD Millipore, Billerica, MA, USA). After blocking, the membranes were probed with antibodies against monoclonal anti-rabbit human E-cadherin (1:1,000; cat. no. 3195), monoclonal anti-rabbit human vimentin $(1: 1,000$; cat. no. 5741) or anti-rabbit human GAPDH $(1: 1,000$; cat. no. 2118; Cell Signaling Technology, Inc., Danvers, MA, USA), or monoclonal anti-mouse human matrix metalloproteinase (MMP-9) antibody (1:800; cat. no. ab58803; Abcam, Cambridge, UK) overnight at $4^{\circ} \mathrm{C}$. After washing in Tris-buffered saline containing Tween 20 (TBST), the membranes of E-cadherin, vimentin and GAPDH were incubated with bovine anit-rabbit horseradish peroxidase-conjugated secondary antibody (1:8,000; cat. no. sc-2370; Santa Cruz Biotechnology, Inc., Dallas, TX, USA) and MMP-9 membranes were incubated with bovine anti-mouse horseradish peroxidase-conjugated secondary antibody (1:8,000; cat. no. sc2371; Santa Cruz Biotechnology, Inc.) at $37^{\circ} \mathrm{C}$ for $1 \mathrm{~h}$. After washing of the membranes in TBST, the proteins were visualized using an enhanced chemiluminescence detection kit (EMD Millipore) and specific antibody binding was visualized and quantified using Image J software (version 1.46; National Institutes of Health, Bethesda, MD, USA). Experiments were performed three times. 
A

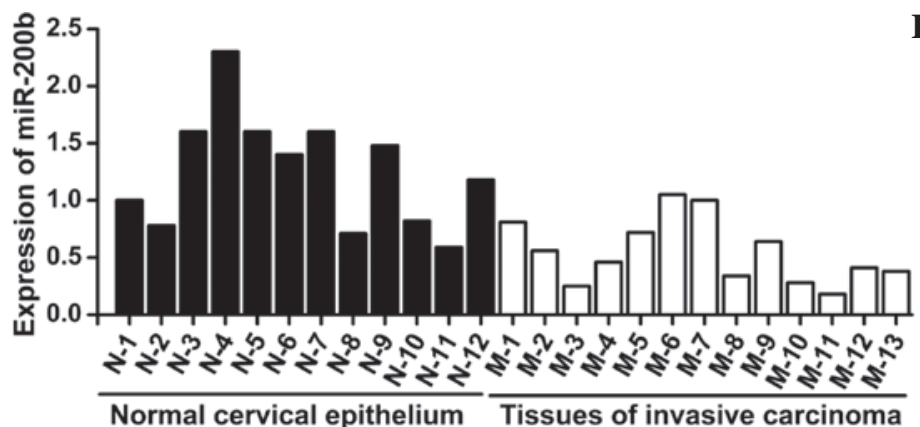

B

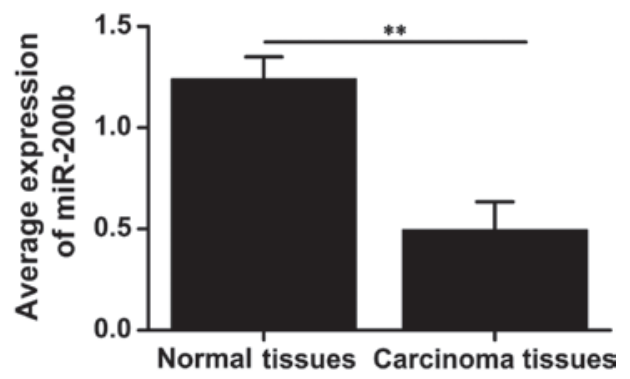

Figure 1. Expression of miR-200b in normal cervical epithelium and invasive carcinoma tissues of the cervix. (A) Expression of miR-200b in normal and cancer tissue specimens from each patient was detected by reverse-transcription quantitative polymerase chain reaction analysis. (B) Average miR-200b expression in normal and carcinoma tissues of the cervix. Values are expressed as the mean \pm standard deviation. ${ }^{* *} \mathrm{P} \leq 0.01$. N/M-1, normal cervical epithelial/cervical carcinoma sample from patient 1 .

Immunofluorescence analysis. Cells grown on coverslips in a culture plate were washed three times with cold PBS and fixed with $4 \%$ paraformaldehyde for $15 \mathrm{~min}$. Coverslips were then washed three times with PBS and rinsed with $0.5 \%$ Triton X-100 in PBS for $20 \mathrm{~min}$. After blocking with goat serum (Goodbio Technology) for $30 \mathrm{~min}$ at room temperature, cells were incubated with primary antibody overnight at $4{ }^{\circ} \mathrm{C}$. Coverslips were rinsed three times with PBS containing Tween 20 (PBST) and then labeled with the secondary antibody for $1 \mathrm{~h}$ in the dark at room temperature. After incubation, coverslips were then washed three times with PBST and incubated with DAPI (Beyotime Institute of Biotechnology) for $5 \mathrm{~min}$ in the dark and washed again with PBST. Following mounting with Antifade Mounting Medium (EMD Millipore), images were acquired with a fluorescence microscope (CKX31; Olympus Corp., Tokyo, Japan).

Statistical analysis. Statistical analyses were performed with SPSS 19.0 software (International Business Machines, Armonk, NY, UA). Values are expressed as the mean \pm standard deviation of at least three independent experiments. The normality test was performed using the K-S test and the homogeneity test for variance was conducted by Levene's test. Statistical analysis was performed using the independent-samples $t$-test (normal distribution, equal variances) and one-way analysis of variance followed by the least-significant differences test (equal variances) or Dunnett's T3 test (unequal variances) when appropriate. $\mathrm{P}<0.05$ was considered to indicate a statistically significant difference between values.

\section{Results}

miR-200b is downregulated in human ICC. To investigate the expression of miR-200b in the cervical cancer tissues and normal adjacent tissues, RT-qPCR was performed on 13 tumor specimens and 12 normal cervical epithelial samples. While miR-200b was expressed in all samples (Fig. 1A), the ICC tissues exhibited a significantly lower expression of miR-200b compared with that in normal tissues $(t=7.353 ; \mathrm{P}=0.002)$ (Fig. 1B), suggesting that miR-200b may have a tumor suppressor role in ICC.

Effects of the transfection of miR-200b mimics or inhibitors on miR-200b expression. For loss-and gain-of-function studies of miR-200b in ICC, HeLa cells were transfected with

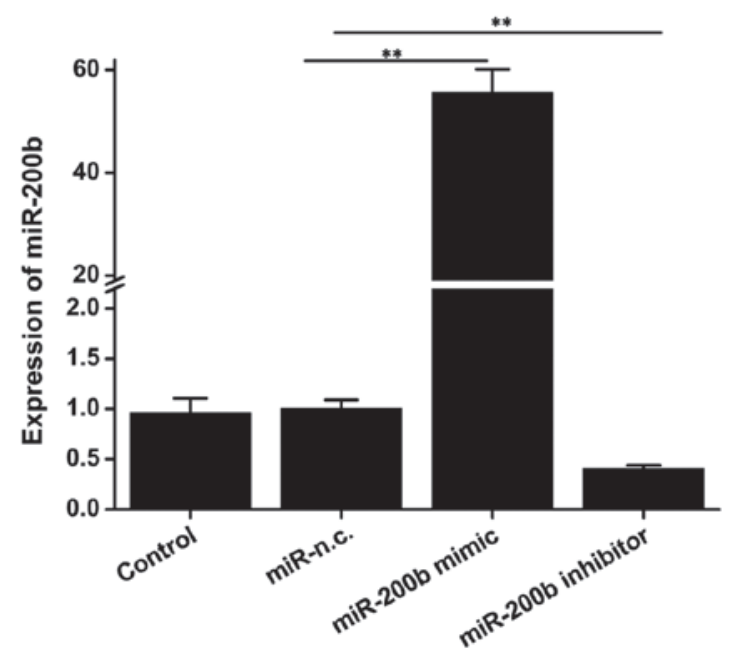

Figure 2. Effects of the transfection of miR-200b mimics and inhibitor on miR-200b levels. Hela cells were transfected with miR-200b mimic and miR-200b inhibitor. Reverse-transcription quantitative polymerase chain reaction analysis was performed. Values are expressed as the mean \pm standard deviation. ${ }^{* *} \mathrm{P} \leq 0.01$. miR, microRNA; n.c., negative control.

miR-200b mimics and inhibitor, and the resulting upregulation or knockdown of miR-200b gene expression were confirmed by RT-qPCR. Transfection of miR-200b mimics significantly increased the expression of miR-200b to 50 fold of that of the negative control group ( $\mathrm{P}=0.007)$ (Fig. 2), while a significant decrease by 0.4 -fold was achieved in cells transfected with inhibitor $(\mathrm{P}=0.01)$. These results suggested the successful overexpression and knockdown, respectively, of miR-200b.

$m i R-200 b$ exerts an anti-migratory effect on ICC. The migratory capacity of neoplastic cells is linked with their degree of malignancy due to its association with metastasis and represents an important potential therapeutic target. Therefore, the present study examined the ability of HeLa cells transfected with miR-200b mimics or miR-200b inhibitor to migrate to the lower compartment. As shown in Fig. 3, transfection with miR-200b mimics significantly inhibited the cell migratory ability compared with that of the negative control group $(\mathrm{P}<0.001)$. Conversely, a significant elevation of the cell migratory ability was found in cells transfected with miR-200b inhibitor $(\mathrm{P}<0.001)$. These results suggested that miR-200b has 


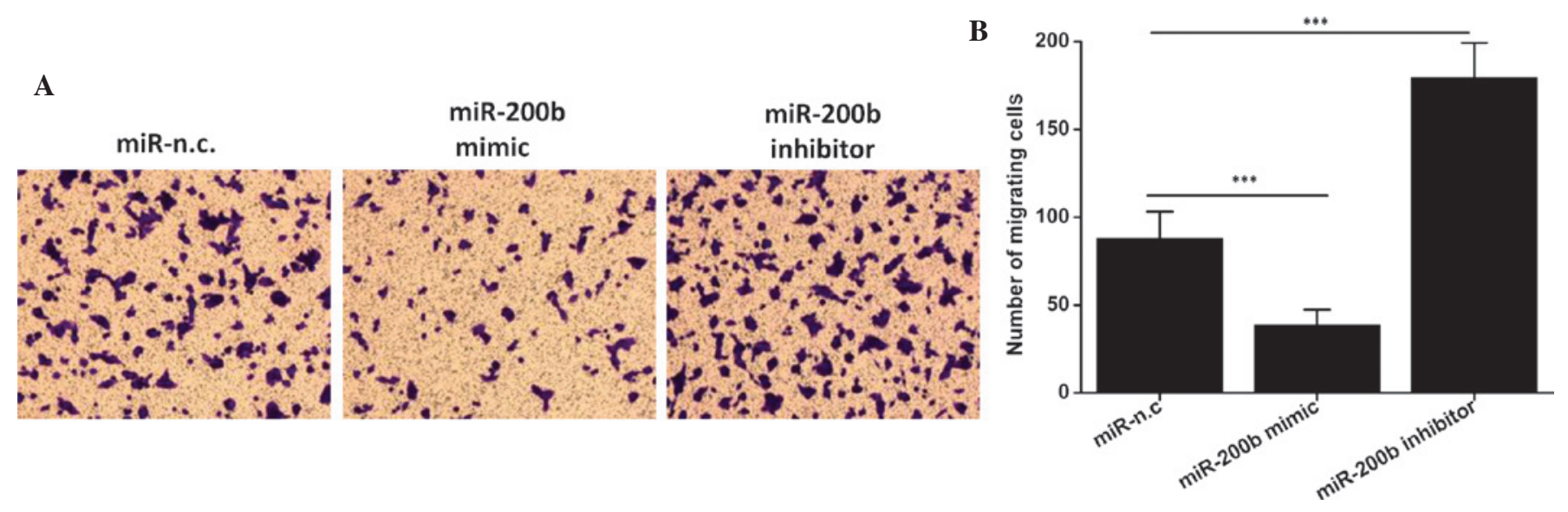

Figure 3. miR-200b exerts an anti-migratory effect on cervical invasive carcinoma cells. (A) Cells were subjected to a Transwell invasion assay. Representative microscopic images from the invasion assay are shown for the three groups (magnification, x100; crystal violet stain). (B) Mean numbers of cells per field migrated to the lower side of the membrane expressed as a percentage of the control. Values are expressed as the mean \pm standard deviation. ${ }^{* *} \mathrm{P} \leq 0.01$. miR, microRNA; n.c., negative control.
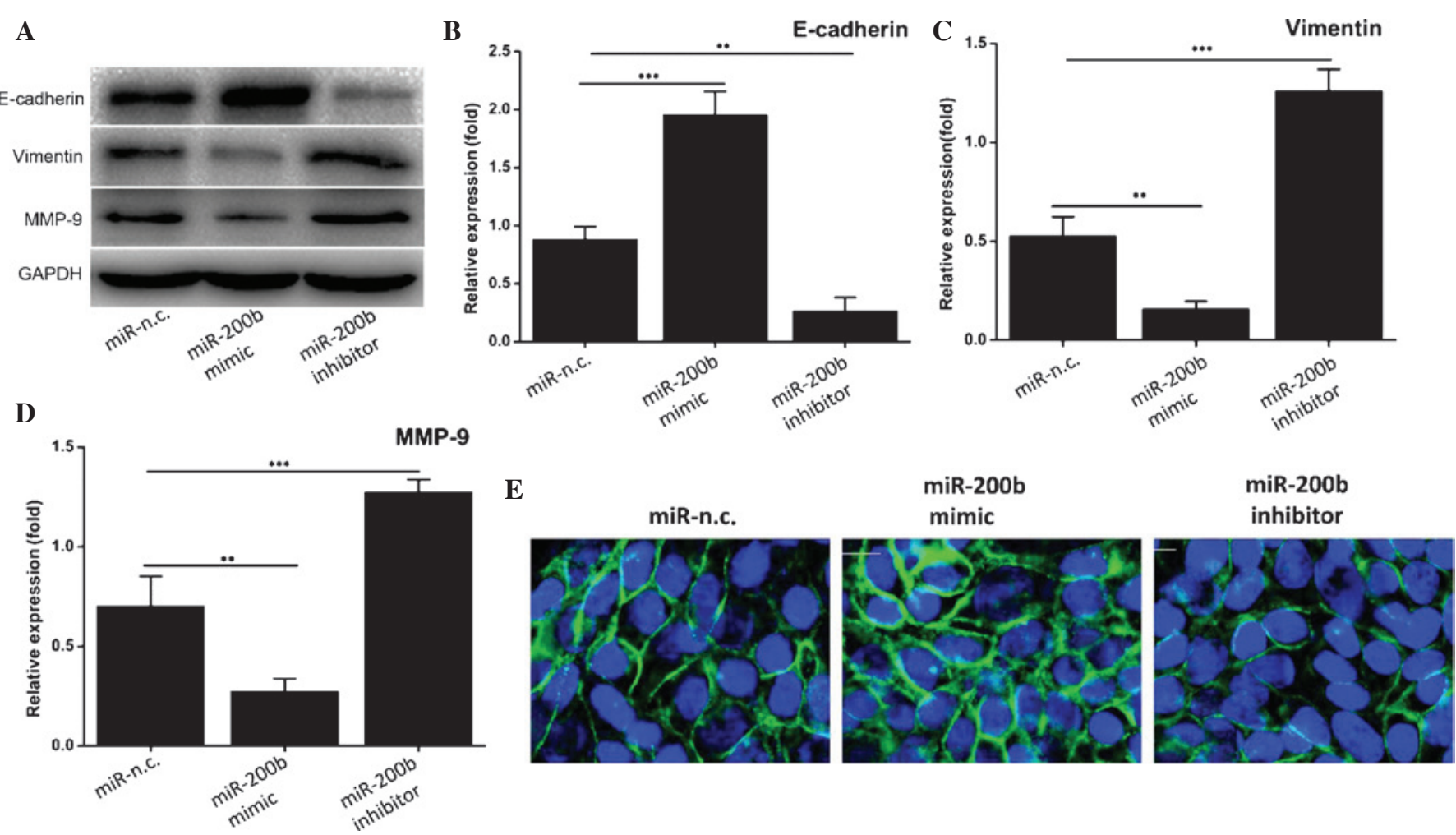

Figure 4. miR-200b suppresses the process of epithelial-mesenchymal transition of cervical carcinoma cells. (A) Western blot analysis of the levels of E-cadherin, vimentin and MMP-9. GAPDH was used as a loading control. A representative blot of three experiments with similar results is shown. (B-D) The expression of E-cadherin, vimentin and MMP-9, respectively, was densitometrically quantified and normalized to GAPDH. Values are expressed as the mean \pm standard deviation $(n=3) .{ }^{* *} \mathrm{P} \leq 0.01,{ }^{* * *} \mathrm{P} \leq 0.001$. (E) The expression of E-cadherin (green) was determined by immunofluorescence analysis. Nuclei were counterstained with DAPI (blue). Magnification, x400. MMP, matrix metalloproteinase; n.c., negative control; GAPDH, glyceraldehyde-3-phosphate dehydrogenase; miR, microRNA.

an inhibitory effect on the migratory ability of ICC, explaining for the downregulation of this tumor suppressor miR in this invasive cancer type.

miR-200b suppresses the EMT of cervical carcinoma cells. To test whether miR-200b affects the expression of proteins involved in the EMT, HeLa cells were transfected with miR-200b mimics or inhibitor. In cells transfected with miR-200b mimics, a substantial elevation of E-cadherin
$(\mathrm{P}<0.001)$ and a reduction in vimentin $(\mathrm{P}=0.002)$ and MMP-9 $(\mathrm{P}=0.002)$ was observed compared to that in the negative control group (Fig. 4A-D). Conversely, inhibition of miR-200b reduced the expression of E-cadherin $(\mathrm{P}=0.002)$, but increased the expression of vimentin $(\mathrm{P}<0.001)$ and MMP-9 $(\mathrm{P}<0.001)$ (Fig. 4A-D). These results indicated that miR-200b was able to suppress the EMT process of HeLa cells, and to therefore potentially inhibit cervical carcinoma cell metastasis. To further verify the effects of miR-200b on the EMT process 
of cervical carcinoma cells, the expression of E-cadherin, which mediates cell-cell adhesion and has a pivotal role in epithelial cell behavior and tissue morphogenesis/remodelling, was determined by immunofluorescence analysis. As shown in Fig. 4E, miR-200b mimics elevated the expression of E-cadherin, while inhibition of miR-200b reduced E-cadherin. These findings provided further evidence to support the causal role of miR-200b in the EMT process of cervical carcinoma.

\section{Discussion}

A large number of miRs have expression patterns that are unique within certain types of cancer tissue (23). It has been suggested that these miRs are potential biomarkers and powerful diagnostic tools for tumor classification and diagnosis $(23,24)$. The aberrant expression of certain miRs in cervical carcinoma is associated with the proliferation, apoptosis, invasion and metastasis of tumor cells (3-5). miR-200b expression has been shown to be decreased in various tumor types, including meningeoma, nasopharyngeal carcinoma, anaplastic thyroid carcinoma and ovarian cancer $(24,25)$. Therefore, the present study assessed miR-200b expression in early ICC by RT-qPCR. miR-200b was found to be downregulated in ICC tissues compared with that in normal cervical epithelium, suggesting that miR-200b may be involved in tumor cell metastasis and invasion. Furthermore, HeLa cells were used to explore the role of miR-200b in cervical carcinoma progression and the underlying molecular mechanisms by transfecting them with miR-200b mimics, inhibitor or negative control miR. A Transwell assay revealed that cells transfected with miR-200b mimics exhibited a reduced migratory potential compared with that of cells transfected with negative control miR, while inhibition of miR-200b enhanced cell migration. This result suggested that miR-200b is involved in the migration and metastasis of HeLa cells.

EMT is a critical process in embryogenesis and a particularly important process during early tumor invasion and metastasis. EMT facilitates the metastasis and progression of tumors by enhancing the migratory and invasive potential of cells (17). Metastatic tumor cells show characteristics of mesenchymal cells as a result of EMT. During cancer progression, transcriptional E-cadherin reprogramming in epithelial cells leads to EMT with decreased adhesion and enhanced migration/invasion. The present study found that overexpression of miR-200b in HeLa cells led to the upregulation of the epithelial marker E-cadherin and downregulation of the mesenchymal marker vimentin, while inhibition of miR-200b had the opposite effects. These results suggested that miR-200b inhibits cervical carcinoma cell metastasis by suppressing EMT. Reduction of E-cadherin expression is a crucial step during EMT. In the present study, immunofluorescence microscopy indicated that E-cadherin expression in HeLa cells was increased following transfection with miR-200b mimics, while it was decreased following miR-200b inhibition. It is known that miR-200 family members regulate EMT by targeting zinc-finger E-box binding homeobox (ZEB) proteins, particularly ZEB1 and ZEB2, and that ZEB2 inhibits the transcription of proteins including E-cadherin and cytokeratin by combining with the E-cadherin gene promoter at the E-box sequence (26-28). ZEB1 and ZEB2 are involved in the EMT through the transforming growth factor- $\beta$ (29), nuclear factor- $\kappa \mathrm{B}$ (30) and Notch (31) pathways. The degradation of extracellular matrix and basement membrane are key steps in tumor invasion and metastasis, and MMP-9 has a central role in this process (32). The present study revealed that overexpression of miR-200b inhibited the expression of MMP-9, leading to the suppression of cervical carcinoma cell metastasis, while the miR-200b inhibitor had the opposite effect. These results further confirmed the involvement of miR-200b in the regulation of the EMT of cervical cancer cells.

In conclusion, the present study demonstrated that miR-200b has a tumor suppressor role in cervical carcinoma. miR-200b was significantly downregulated in ICC tissues compared with normal adjacent tissues. Upregulation of miR-200b in HeLa cells reduced their migratory potential and inhibited EMT, as indicated by enhanced E-cadherin expression and reduced vimentin and MMP-9 expression, while inhibition of miR-200b had the opposite effect. These results indicated that miR-200b may be able to inhibit the progression and metastasization of cervical cancer. miR-200b mimics may therefore be utilized for targeting of cervical carcinoma and other tumor types.

\section{Acknowledgements}

The present study was supported by the Natural Science Foundation of China (nos. 81302273 and 81201196), the Science and Technology Department of Hubei Province, China (no. ZRY039), the Health and Family Planning Commission of Hubei Province, China (no. 2012ZY02) and the Chinese Postdoctoral Science Foundation (no. 2011M500857).

\section{References}

1. Cai HB, Ding XH and Chen CC: Prevalence of single and multiple human papillomavirus types in cervical cancer and precursor lesions in Hubei, China. Oncology 76: 157-161, 2009.

2. Granados López AJ and López JA: Multistep model of cervical cancer: Participation of miRNAs and coding genes. Int J Mol Sci 15: 15700-15733, 2014.

3. Kloosterman WP and Plasterk RH: The diverse functions of microRNAs in animal development and disease. Dev Cell 11: 441-450, 2006.

4. Kestens C, Siersema PD and van Baal JW: Current understanding of the functional roles of aberrantly expressed microRNAs in esophageal cancer. World J Gastroenterol 22: 1-7, 2016.

5. Frixa T, Donzelli S and Blandino G: Oncogenic microRNAs: Key players in malignant transformation. Cancers (Basel) 7: 2466-2485, 2015.

6. O'Day E and Lal A: MicroRNAs and their target gene networks in breast cancer. Breast Cancer Res 12: 201, 2010.

7. Lim YY, Wright JA, Attema JL, Gregory PA, Bert AG, Smith E, Thomas D, Lopez AF, Drew PA, Khew-Goodall Y and Goodall GJ: Epigenetic modulation of the miR-200 family is associated with transition to a breast cancer stem-cell-like state. J Cell Sci 126: 2256-2266, 2013.

8. Schliekelman MJ, Gibbons DL, Faca VM, Creighton CJ, Rizvi ZH, Zhang Q, Wong CH, Wang H, Ungewiss C, Ahn YH, et al: Targets of the tumor suppressor miR-200 in regulation of the epithelial-mesenchymal transition in cancer. Cancer Res 71: 7670-7682, 2011.

9. Iliopoulos D, Lindahl-Allen M, Polytarchou C, Hirsch HA, Tsichlis PN and Struhl K: Loss of miR-200 inhibition of Suz12 leads to polycomb-mediated repression required for the formation and maintenance of cancer stem cells. Mol Cell 39: 761-772, 2010.

10. Uhlmann S, Zhang JD, Schwäger A, Mannsperger H, Riazalhosseini Y, Burmester S, Ward A, Korf U, Wiemann S and Sahin O: miR-200bc/429 cluster targets PLCgamma1 and differentially regulates proliferation and EGF-driven invasion than miR-200a/141 in breast cancer. Oncogene 29: 4297-4306, 2010. 
11. Schickel R, Park SM, Murmann AE and Peter ME: miR-200c regulates induction of apoptosis through CD95 by targeting FAP-1. Mol Cell 38: 908-915, 2010.

12. Magenta A, Cencioni C, Fasanaro P, Zaccagnini G, Greco S Sarra-Ferraris G, Antonini A, Martelli F and Capogrossi MC: miR-200c is upregulated by oxidative stress and induces endothelial cell apoptosis and senescence via ZEB1 inhibition. Cell Death Differ 18: 1628-1639, 2011.

13. Yang $J$ and Weinberg RA: Epithelial-mesenchymal transition: At the crossroads of development and tumor metastasis. Dev Cell 14: 818-829, 2008

14. Thompson EW, Newgreen DF and Tarin D: Carcinoma invasion and metastasis: A role for epithelial-mesenchymal transition? Cancer Res 65: 5991-5995, 2005.

15. von Burstin J, Eser S, Paul MC, Seidler B, Brandl M, Messer M, von Werder A, Schmidt A, Mages J, Pagel P, et al: E-cadherin regulates metastasis of pancreatic cancer in vivo and is suppressed by a SNAIL/HDAC1/HDAC2 repressor complex. Gastroenterology 137: 361-371.371.e1-e5, 2009

16. Haslehurst AM, Koti M, Dharsee M, Nuin P, Evans K, Geraci J, Childs T, Chen J, Li J, Weberpals J, et al: EMT transcription factors snail and slug directly contribute to cisplatin resistance in ovarian cancer. BMC Cancer 12: 91, 2012.

17. Hanahan D and Weinberg RA: Hallmarks of cancer: The next generation. Cell 144: 646-674, 2011.

18. Kang Y and Massagué J: Epithelial-mesenchymal transitions: Twist in development and metastasis. Cell 118: 277-279, 2004.

19. Lamouille S, Xu J and Derynck R: Molecular mechanisms of epithelial-mesenchymal transition. Nat Rev Mol Cell Biol 15: 178-196, 2014

20. Chen Y, Xiao Y, Ge W, Zhou K, Wen J, Yan W, Wang Y, Wang B, Qu C, Wu J et al: miR-200b inhibits TGF- $\beta 1$-induced epithelial-mesenchymal transition and promotes growth of intestinal epithelial cells. Cell Death Dis 4: e541, 2013

21. Pecorelli S. Revised FIGO staging for carcinoma of the vulva, cervix, and endometrium. Int J Gynaecol Obstet. 105: 103-104, 2009.

22. Livak KJ and Schmittgen TD: Analysis of relative gene expression data using real-time quantitative PCR and the 2(-Delta Delta C(T)) Method. Methods 25: 402-408, 2001
23. Lim J and Thiery JP: Epithelial-mesenchymal transitions: Insights from development. Development 139: 3471-3486, 2012.

24. Vergara D, Merlot B, Lucot JP, Collinet P, Vinatier D, Fournier I and Salzet M: Epithelial-mesenchymal transition in ovarian cancer. Cancer Lett 291: 59-66, 2010.

25. Park SM, Gaur AB, Lengyel E and Peter ME: The miR-200 family determines the epithelial phenotype of cancer cells by targeting the E-cadherin repressors ZEB1 and ZEB2. Genes Dev 22: 894-907, 2008.

26. Burk U, Schubert J, Wellner U, Schmalhofer O, Vincan E, Spaderna $\mathrm{S}$ and Brabletz T: A reciprocal repression between ZEB1 and members of the miR-200 family promotes EMT and invasion in cancer cells. EMBO Rep 9: 582-589, 2008.

27. Gregory PA, Bert AG, Paterson EL, Barry SC, Tsykin A, Farshid G, Vadas MA, Khew-Goodall Y and Goodall GJ: The miR-200 family and miR-205 regulate epithelial to mesenchymal transition by targeting ZEB1 and SIP1. Nat Cell Biol 10: 593-601, 2008.

28. Korpal M,Lee ES,Hu G and Kang Y: The miR-200 family inhibits epithelial-mesenchymal transition and cancer cell migration by direct targeting of E-cadherin transcriptional repressors ZEB1 and ZEB2. J Biol Chem 283: 14910-14914, 2008.

29. Postigo AA: Opposing functions of ZEB proteins in the regulation of the TGFbeta/BMP signaling pathway. EMBO J 22: 2443-2452, 2003.

30. Chua HL, Bhat-Nakshatri P, Clare SE, Morimiya A, Badve S and Nakshatri H: NF-kappaB represses E-cadherin expression and enhances epithelial to mesenchymal transition of mammary epithelial cells: Potential involvement of ZEB-1 and ZEB-2. Oncogene 26: 711-724, 2007.

31. Wang Z, Li Y, Kong D, Banerjee S, Ahmad A, Azmi AS, Ali S, Abbruzzese JL, Gallick GE and Sarkar FH: Acquisition of epithelial-mesenchymal transition phenotype of gemcitabine-resistant pancreatic cancer cells is linked with activation of the notch signaling pathway. Cancer Res 69: 2400-2407, 2009.

32. Xu E, Xia X, Lü B, Xing X, Huang Q, Ma Y, Wang W and Lai M: Association of matrix metalloproteinase-2 and -9 promoter polymorphisms with colorectal cancer in Chinese. Mol Carcinog 46: 924-929, 2007 